\title{
Replik zu «Eine Professur für Pallia- tivmedizin in der Radio-Onkologie?»
}

\author{
Matthias Guckenberger ${ }^{a}$, Jürg Hodler ${ }^{b}$, Rainer Weber ${ }^{c}$

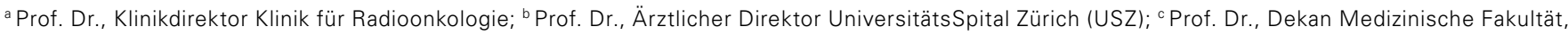 \\ Universität Zürich (UZH)
}

Herr Prof. Borasio adressiert mit der Palliativmedizin ein interdisziplinäres Querschnittsfach, das sich in den letzten Jahren durch den gemeinsamen Einsatz und das Engagement von Politik, Fachverband, universitären und ausseruniversitären Einrichtungen in der klinischen Versorgung, Lehre und Forschung erfreulich entwickelt hat. Wir teilen die Überzeugung, dass die bisherigen Erfolge noch nicht ausreichend sind und weitergehende Anstrengungen notwendig sind.

\section{Das Kompetenzzentrum Palliative Care des USZ} betreibt mit 12 Betten für spezialisierte Palliative Care die grösste universitäre Station in der Schweiz.

Daher haben das UniversitätsSpital Zürich (USZ) sowie die Universität Zürich (UZH) gezielt und strategisch in dieses Thema investiert. Das Kompetenzzentrum Palliative Care des USZ betreibt mit 12 Betten für spezialisierte Palliative Care die grösste universitäre Station in der Schweiz, ein weiterer Ausbau auf 16 Betten ist für Q3 2018 geplant. Die spezialisierte Palliative Care ist über Konsiliardienst und Sprechstunde breit nach USZ-intern sowie nach ambulant vernetzt. Im Stadium eines Pilotprojekts befindet sich der Aufbau eines interdisziplinären und multiprofessionellen Spital-weiten Netzwerks zur Förderung von allgemeiner und spezialisierter Palliative Care. In der Lehre ist die Palliativmedizin sowohl im obligatorischen Curriculum als auch im vertiefend im Mantelstudium verankert; in Zusammenarbeit mit dem Lehrstuhl für Spiritual Care der theologischen Fakultät haben wir interprofessionelle Lehre über die Grenzen der medizinischen Fakultät etabliert. Von der SAMW wird innovative Forschung in Kooperation mit der ETH Zürich, Wearable Computing Lab, gefördert, und erste vielversprechende Ergebnisse sind erfolgreich publiziert.

1 Borasio GD. Eine Professur für Palliativmedizin in der Radio-Onkologie? Schweiz Ärztezeitung. 2018. 99(17):553-4.
Unterstützung der Margrit Weisheit Stiftung ermöglicht, für die UZH und das USZ der Stiftung dankbar sind. Die Professur hat die Ziele, die Palliativmedizin breiter im Studium zu verankern und die Forschungsaktivitäten auszubauen.

Der Brief des Kollegen Borasio scheint zum Teil auf Unkenntnis der Situation am USZ und der UZH zu beruhen. Geäussert werden Bedenken über eine angebliche Fokussierung der Palliative Care auf onkologische Patienten. Das Kompetenzzentrum Palliative Care wird erfolgreich durch ein multiprofessionelles und insbesondere interdisziplinäres Leitungsgremium geführt: Die Einbindung von Radioonkologie, Onkologie, Neurologie, Kardiologie und Dermatologie garantiert eine optimale Entwicklung der Palliative Care, um den Herausforderungen einer alternden Bevölkerung mit den drei Haupttodesursachen - Krebs, kardiovaskuläre und neurodegenerative Erkrankungen - gerecht zu werden. Die Ansiedlung in Radioonkologie ist tatsächlich historisch gewachsen, es handelt sich um eine strukturelle Anbindung, und nicht um eine inhaltliche Fixierung. Einen umsichtigen Einsatz von Ressourcen und die Suche nach Synergien zwischen Fächern und Disziplinen erachten wir als essentiell, da gerade die Tarif-

Wir haben am USZ daher aktiv die Entscheidung getroffen, zum direkten Wohle unserer Patienten in klinische Strukturen sowie ärztliches und Pflegepersonal zu investieren, statt in administrativen Overhead.

situation in der Palliative Care immer noch unbefriedigend ist. Im Gegensatz zu anderen Kantonen erfolgt in Zürich keine Subventionierung der universitären Medizin durch die Politik. Wir haben am USZ daher aktiv die Entscheidung getroffen, zum direkten Wohle unserer Patienten in klinische Strukturen sowie ärztliches und Pflegepersonal zu investieren, statt in administrativen Overhead.

Ebenso scheint beim Autor Unkenntnis über das Vorlesungsverzeichnis des Humanmedizinstudiums der UZH zu bestehen. Lehrveranstaltungen zum Thema 
* Deutsche Krebsgesellschaft

Korrespondenz: Prof. Dr. Matthias Guckenberger Klinikdirektor Klinik für Radioonkologie UniversitätsSpital Zürich Rämistrasse 100 CH-8091 Zürich Tel 0442552930 Matthias.Guckenberger[at] usz ch
Palliativmedizin haben kein "Label RadioOnkologie», die Lehre ist unabhängig von Kliniken und Instituten in Form von interdisziplinären Themenblöcken organisiert. Wir möchten zudem betonen, dass alle Professuren, auch Assistenzprofessuren, der UZH unabhängige Forschung und Lehre betreiben.

Der Paragraph über eine «Domestizierung» der Palliative Care hat bei uns Unverständnis ausgelöst. Zwischen den Zeilen wird mehr oder weniger verdeckt der Vorwurf formuliert, ein Lehrstuhl für Palliativmedizin sei notwendig, um unsere Patienten vor der Profitgier der Onkologie zu schützen. Wir empfinden dies als Diskreditierung aller engagierter Palliativmediziner/innen, die auch ohne den Status eines Ordinarius hervorragende klinische Arbeit leisten, an universitären wie auch nicht-universitären Einrichtungen. Wir empfinden dies ebenso als eine Diskreditierung der universitären Onkologie mit ihren evidenzbasierten, interdisziplinär abgestimmten und den Patientenwunsch achtenden Entscheidungsprozessen, mit selbstverständlicher Berücksichtigung palliativmedizinischer Grundsätze. Die Onkologie hat hier tatsächlich eine Vorreiterrolle, indem Palliativ Care essentieller Bestandteil DKG*-zertifizierter Krebszentren ist.

Die universitäre Medizin und Forschung werden in $\mathrm{Zu}$ kunft vermehrt auf die finanzielle Unterstützung durch Stiftungen und engagierte Bürger/innen angewiesen sein. Wir sind der Margrit Weisheit Stiftung daher sehr dankbar für ihren grosszügigen Beitrag, die Palliativmedizin am Standort Zürich akademisch zu etablieren. Wir sind überzeugt, damit den Grundstein für eine nachhaltige akademische Entwicklung der Palliativmedizin zu legen. Berufspolitisch motivierte Aktionen bergen die Gefahr, dieses öffentliche Engagement zu beschädigen. Die UZH hatte bereits zu Beginn

\section{Die universitäre Medizin und Forschung} werden in Zukunft vermehrt auf die finanzielle Unterstützung durch Stiftungen und engagierte Bürger/innen angewiesen sein.

des Berufungsprozesses Herrn Prof. Borasio eingeladen, aktiv an der Entwicklung der akademischen Palliativmedizin in Zürich mitzuwirken, was er aber abgelehnt hatte. Wir hoffen mit dieser Replik Klarheit geschaffen zu haben, dass das USZ und die UZH die Palliativmedizin stärken wollen: in der Betreuung unserer Patientinnen und Patienten, in Forschung und Lehre wie auch modellhaft in der interprofessionellen und interdisziplinären Zusammenarbeit. 